\title{
Crecimiento y desarrollo intestinal de aves de engorde alimentadas con cepas probióticas
}

\author{
Chávez, L.A.; López, A. y Parra, J.E.@
}

Grupo Biodiversidad y Genética Molecular BIOGEM. Departamento de Producción Animal. Facultad de Ciencias Agrarias. Universidad Nacional de Colombia. Medellín. Colombia.

\section{PALABRAS CLAVE ADICIONALES}

Aditivos.

Aves de corral.

Bacterias ácido lácticas.

Sistema digestivo.

Vellosidades intestinales.

\section{RESUMEN}

A través de la alimentación, los animales se exponen a agentes extraños, donde el epitelio intestinal actúa como una barrera natural contra las bacterias y sustancias tóxicas que estén presentes en el lumen intestinal. No obstante, la ingesta de bacterias probióticas podría influir sobre el desarrollo y función de órganos digestivos, específicamente el intestino, mejorando los parámetros fisiológicos, nutricionales e inmunológicos de este. El objetivo de este trabajo fue evaluar diferentes cepas probióticas sobre el crecimiento alométrico y desarrollo intestinal de pollos de engorde durante su etapa productiva. Se utilizaron 125 pollos machos (Cobb) de un día de edad y alimentados con dos dietas: dieta comercial con y sin la adición de antibiótico. Los diferentes probióticos (Lactobacillus casei, Lactobacillus acidophilus ó Enterococcus faecium) se suministraron en el agua de bebida de los animales que consumieron la dieta basal sin antibiótico garantizando una concentración de $10^{7} \mathrm{UFC} / \mathrm{ml}$. El diseño estadístico utilizado fue de bloques al azar en arreglo de parcelas divididas. La inclusión de probióticos, específicamente $E$. faecium, en la alimentación de pollos de engorde mejoraron el peso, desarrollo y crecimiento de órganos de importancia digestiva, específicamente intestino, lo cual se ve reflejado en vellosidades con mayor altura y ancho, y criptas menos profundas $(p<0,01)$; lo que podría mejorar la absorción de nutrientes y por consiguiente la salud de los animales. Por lo anterior, E. faecium, puede ser utilizado en la alimentación de aves durante todo el ciclo productivo como promotor de crecimiento.

\section{Intestinal growth and development of broilers fed with probiotic strains}

\section{SUMMARY}

Through feeding, animals are exposed to foreign agents, in which the intestinal epithelium acts as a natural barrier against bacteria and toxic substances which may be present in the intestinal lumen. However, probiotic bacteria intake could influence the development and function of digestive organs, specifically the intestine, improving its physiological, nutritional and immunological parameters. The goal of this study was to evaluate different probiotic strains on broilers' allometric growth and intestinal development during their productive stages. 125 one-day-old male chicks (Cobb) were used and fed on two diets: Commercial diet with and without antibiotic addition. Different probiotics (Lactobacillus casei, Lactobacillus acidophilus ó Enterococcus faecium) were supplied in the drinking water of the animals fed on the basal diet without antibiotic ensuring a concentration of $10^{7} \mathrm{UFC} / \mathrm{ml}$. The statistical design used was a randomized split-plot arrangement. The inclusion of probiotics, specifically E. faecium, in broiler feed improved important digestive organs weight, development and growth, particularly the intestine, which is reflected in greater villus height and width, and less deep crypts ( $<<0.01$ ); potentially improving nutrient absorption and therefore the health of the animals. Therefore, E. faecium, can be used in bird feeding throughout the production cycle as a growth promoter.
INFORMACIÓN

\section{Cronología del artículo.}

Recibido/Received: 04.08.2015

Aceptado/Accepted: 16.11.2015

On-line: 16.03 .2016

Correspondencia a los autores/Contact e-mail:

jeparrasu@unal.edu.co

\section{INTRODUCCIÓN}

La avicultura es una actividad que ha alcanzado grandes avances en las últimas décadas, y esto se debe principalmente a la acción conjunta entre genética, sanidad, manejo y nutrición (Kalmar et al., 2013). Desde el punto de vista nutricional, los animales se exponen a agentes extraños a través de los diferentes alimentos utilizados, los cuales en un momento determinado podrían ocasionar una reacción inmunológica (Giannenas et al., 2012; Korver, 2012). 
El epitelio intestinal actúa como una barrera natural contra las bacterias patógenas y sustancias tóxicas que están presentes en el alimento y lumen intestinal. Algunos de estos factores pueden causar alteraciones en la microbiota normal y/o en el epitelio intestinal alterando la permeabilidad de esta, facilitando la invasión de patógenos y sustancias perjudiciales, las cuales provocan la aparición de procesos inflamatorios crónicos, y a su vez, la disminución en el tamaño de las vellosidades, y en los procesos de digestión y absorción de nutrientes (Lodemann, 2010; Chambers and Gong, 2011; Plaza et al., 2014).

Para disminuir la aparición de estos problemas, en los últimos años se venían utilizando en la alimentación animal antibióticos como promotores de crecimiento (APC) en dosis subterapéuticas; los cuales no sólo influyen en las poblaciones microbianas intestinales y sus actividades, sino que además, afectan el metabolismo de los animales y alteran específicamente la función intestinal (Huyghebaert et al., 2011; Král et al., 2012). En la actualidad, la preocupación mundial sobre el desarrollo de la resistencia a los antimicrobianos y sobre la transferencia de genes de resistencia a los antibióticos de los animales a la microbiota humana, condujo a la prohibición del uso de antibióticos como promotores del crecimiento en la Unión Europea desde el 1 de enero 2006 (Gaggìa et al., 2010).

Por tal motivo, como estrategia terapéutica de origen nutricional se ha propuesto el uso de probióticos, ya que se ha reportado que estos podrían mejorar los parámetros productivos, fisiológicos, y nutricionales en algunas especies animales (Quigley, 2010). Por lo anterior, el objetivo de este trabajo fue evaluar diferentes cepas probióticas sobre el crecimiento alométrico y desarrollo intestinal de pollos de engorde durante su etapa productiva.

\section{MATERIAL Y MÉTODOS}

\section{LOCALIZACIÓN}

El trabajo de campo se realizó en la granja comercial, Los Andes, ubicada en el municipio de Girardota (Antioquia, Colombia), vereda El Totumo, localizado a $1425 \mathrm{msnm}$, con una temperatura promedio de $22^{\circ} \mathrm{C}$, correspondiendo a una zona de vida bosque húmedo premontano (bh-PM).

\section{ANIMALES}

Se utilizaron 125 pollos machos de un día de edad de la línea comercial Cobb obtenidos de una casa comercial, con un peso promedio inicial de 45,65 g. El período experimental tuvo una duración de 42 días. La cría se realizó siguiendo los procedimientos comerciales establecidos en la granja.

\section{INSTALACIONES Y EQUIPOS}

Los pollos fueron alojados dentro de un galpón comercial con piso de cemento y con cama de viruta de madera, en el cual se realizaron las divisiones para los tratamientos utilizando cartón plast. Cada división tuvo una medida de 1,0 $\times 1,2$ metros, además de comedero y bebedero independiente. Durante la primera y segunda semana se utilizaron comederos de bandeja, posteriormente se cambió a comedero de tolva. Durante todo el experimento se utilizaron bebederos de volteo de 31 de capacidad, y el agua se suministraba dos veces al día. Para mantener la temperatura homogénea durante las primeras semanas se utilizaron criadoras a gas, y posteriormente cortinas alrededor del galpón para controlar la temperatura, humedad y entrada-salida de corrientes de aire. Para realizar el pesaje de los pollos y el alimento suministrado se utilizó una balanza digital.

\section{MANEJO SANITARIO}

Para la recepción de los pollos, se realizó lavado, limpieza y desinfección del galpón, cortinas, comederos y bebederos; además, se hizo el control de roedores e insectos con productos obtenidos en casas comerciales. Las criadoras se encendieron cinco horas antes de la llegada de los animales.

\section{DIETAS}

Los animales fueron alimentados con dos dietas: dieta comercial con y sin la adición de antibiótico. Los diferentes probióticos (Lactobacillus casei, Lactobacillus acidophilus ó Enterococcus faecium) se suministraron en el agua de bebida de los animales que consumieron la dieta comercial sin antibiótico, así:

- Dieta 1 Control (DC): Alimento comercial sin antibiótico, sin adición de cepa probiótica en el agua de bebida.

- Dieta 2 (D2): Alimento comercial con antibiótico, sin adición de cepa probiótica en el agua de bebida.

- Dieta 3 (D3): Alimento comercial sin antibiótico, con adición de la cepa comercial probiótica Lactobacillus acidophilus en el agua de bebida.

- Dieta 4 (D4): Alimento comercial sin antibiótico, con adición de la cepa comercial probiótica Lactobacillus casei en el agua de bebida.

- Dieta 5 (D5): Alimento comercial sin antibiótico, con adición de la cepa comercial probiótica Enterococcus faecium en el agua de bebida.

Las dietas ofrecidas a los animales cumplieron con los requerimientos mínimos nutricionales establecidos por Rostagno (2011). Se elaboraron 2 dietas (tabla I), una para la etapa de iniciación (día 1 al 21), y la otra para la etapa de finalización (día 22 al 42). El alimento utilizado en el estudio estuvo libre de antibióticos (excepto la dieta D2), ya que no fue de interés modificar la dieta, sino la incorporación de los probióticos como una alternativa al uso de antibióticos. La cantidad de probiótico adicionado se realizó siguiendo las instrucciones para su preparación y adición según lo recomendado por el fabricante. La inclusión de los probióticos en el agua de bebida se realizó por mezclado directo de un litro de agua con 30 gramos de azúcar comercial, para garantizar poblaciones mínimas de $10^{8} \mathrm{UFC}$, con una viabilidad adecuada, la cual fue evaluada por medio de análisis microbiológicos semanales. Las dietas experimentales se proporcionaron durante todo el ciclo productivo (dia 1-42 de vida). 
Tabla I. Composición de la dieta basal (Basal diet composition).

\begin{tabular}{|c|c|c|c|c|}
\hline & $\mathrm{DBI}(\%)$ & $\mathrm{DB}+\mathrm{Al}(\%)$ & $\mathrm{DBF}(\%)$ & $\mathrm{DB}+\mathrm{AF}(\%)$ \\
\hline Maiz & 65,22 & 65,11 & 69,29 & 69,23 \\
\hline Torta de soya & 28,22 & 28,22 & 14,19 & 14,19 \\
\hline Soya frijol cocido & 2,6 & 2,6 & 13 & 13 \\
\hline Carbonato de calcio fino & 1,37 & 1,37 & 1,23 & 1,23 \\
\hline Fosfato monocálcico & 0,99 & 0,99 & 0,8 & 0,8 \\
\hline Aceite de palma & 0,5 & 0,5 & 0,5 & 0,5 \\
\hline Sal fina & 0,33 & 0,33 & 0,33 & 0,33 \\
\hline DL-metionina & 0,18 & 0,18 & 0,14 & 0,14 \\
\hline L-lisina HCL & 0,15 & 0,15 & 0,11 & 0,11 \\
\hline $\begin{array}{l}\text { GFC broiler premix } \\
1.2 \mathrm{P}\end{array}$ & 0,12 & 0,12 & 0,12 & 0,12 \\
\hline Bicarbonato de sodio & 0,1 & 0,1 & 0,08 & 0,08 \\
\hline $\begin{array}{l}\text { Atrapante de micotoxi- } \\
\text { nas (Mycofix secure) }\end{array}$ & 0,09 & 0,09 & 0 & 0 \\
\hline Cloruro de colina $60 \%$ & 0,07 & 0,07 & 0,05 & 0,05 \\
\hline Zinc bacitracina $15 \%$ & 0 & 0,05 & 0 & 0 \\
\hline $\begin{array}{l}\text { Maduramicina } 0,75 \% \text { y } \\
\text { nicarbazina } 8 \%\end{array}$ & 0 & 0,05 & 0 & 0 \\
\hline Sulfato de cobre $25 \%$ & 0,04 & 0,04 & 0,04 & 0,04 \\
\hline Triptófano & 0,02 & 0,02 & 0,01 & 0,01 \\
\hline Neomicina $50 \%$ & 0 & 0,02 & 0 & 0 \\
\hline L-treonina & 0,01 & 0,01 & 0,12 & 0,12 \\
\hline Salinomicina $12 \%$ & 0 & 0 & 0 & 0,05 \\
\hline Enramicina $8 \%$ & 0 & 0 & 0 & 0,01 \\
\hline Colistina $50 \%$ & 0 & 0 & 0 & 0,01 \\
\hline
\end{tabular}

Análisis proximal de las dietas (Proximal analysis of the diets).

\begin{tabular}{lcccc}
\hline EM (kcal/kg) & 2984 & 2984 & 3152 & 3152 \\
PC (\%) & 19,16 & 19,16 & 17 & 17 \\
EE (\%) & 4,047 & 4,047 & 6,023 & 6,023 \\
FC (\%) & 2,742 & 2,742 & 2,748 & 2,748 \\
Humedad (\%) & 10,52 & 10,52 & 10,79 & 10,79
\end{tabular}

$\mathrm{DBI}=$ Dieta basal de iniciación; $\mathrm{DB}+\mathrm{Al}=$ Dieta basal + antibiótico de iniciación; $\mathrm{DBF}=$ Dieta basal de finalización; $\mathrm{DB}+\mathrm{AF}=$ Dieta basal + antibiótico de finalización; EM= Energía metabolizable (kcal $/ \mathrm{kg})$; $\mathrm{PC}=$ Proteína cruda $(\%) ; \mathrm{EE}=$ Extracto etéreo $(\%) ; \mathrm{FC}=$ Fibra cruda $(\%)$

\section{EUTANASIAS HUMANITARIAS Y TOMA DE MUESTRAS ÓRGANOS}

Durante la fase experimental se realizó eutanasia humanitaria a 125 aves de la siguiente forma: El día inicial, o Día 1, se sacrificaron 5 aves que representaron el grupo de referencia para verificar el estado general de salud y la evaluación macroscópica del estado de los órganos antes de suministrar las dietas experimentales; y las unidades experimentales para cada uno de los bloques. Durante la fase de eutanasias escalonadas se sacrificaron 120 aves de la siguiente forma: los días $7,14,21,28,35$ y 42 se sacrificaron 20 aves/día (cuatro aves por tratamiento). Los animales se sedaron por inhalación con Nitrox® y posteriormente se les realizó eutanasia humanitaria con dióxido de carbono durante 3 minutos. Todas las aves fueron sacrificadas 2,5 horas después de su última comida.

Después del sacrificio, las aves se pusieron en posición ventral boca arriba, separando los miembros posteriores y extendiendo las alas lateralmente; luego se realizó un corte que iba desde la parte anterior del cuello hasta la cloaca, intentando cortar solo la piel.
Para la apertura de la cavidad celómica se realizó un corte con las tijeras en la zona que se encuentra por debajo de la pechuga. Se realizaron dos pequeños cortes laterales hasta llegar a las costillas, y luego un corte de las costillas en dirección craneal. En este instante se evaluó la presencia de exudados diversos y el estado de sacos aéreos (Dolz and Majó, 2011).

Los órganos de la cavidad celómica se extrajeron conjuntamente. Se retiró el tracto gastrointestinal con el hígado y el bazo. El proventrículo y la molleja se separaron del intestino y se abrieron con tijera examinando el contenido y la mucosa. Del intestino delgado, se tomaron $20 \mathrm{~cm}$ de todas las porciones (duodeno, yeyuno e íleon); y se tomó una muestra del páncreas que se encuentra adherido al mesenterio duodenal. Todos los órganos extraídos fueron lavados con solución salina fría, y luego fueron pesados para los análisis alométricos (Reis et al., 2005).

\section{ANÁLISIS ALOMÉTRICO}

Los pesos de los órganos fueron convertidos a porcentaje de peso vivo $(\% \mathrm{PV})$ por medio de la fórmula:

$$
\% \mathrm{PV}=\frac{\text { Peso órgano } \times 100}{\text { Peso promedio } / \text { ave }}
$$

Para determinar la ontogénesis del crecimiento de los diferentes órganos y su relación con el peso corporal, se utilizó la constante de Crecimiento alométrico (CA), así:

Donde:

$$
\mathrm{CA}=\frac{\mathrm{O}_{\mathrm{n}} / \mathrm{O}_{\mathrm{h}}}{\mathrm{PC}_{\mathrm{n}} / \mathrm{PC}_{\mathrm{h}}}
$$

$\mathrm{O}=$ peso del órgano;

$\mathrm{n}=$ días después del nacimiento;

$\mathrm{h}=$ peso al nacimiento $\mathrm{y}$

$\mathrm{PC}=$ peso corporal.

Cuando el órgano crece en la misma proporción al peso corporal, CA es de 1; si el crecimiento del órgano es menor al peso corporal, CA es menor a 1; y cuando CA es mayor a 1 , hay un crecimiento rápido en relación con la ganancia total de peso corporal (Cortés and Villamarin, 2013; Jaramillo, 2012).

\section{ANÁLISIS MORFOMÉTRICO DEL INTESTINO DELGADO}

Transcurrido 48 horas después de la colecta, las muestras de las diferentes secciones del intestino almacenadas en formalina al $10 \%$, fueron enviadas al laboratorio de Histopatología Animal de la Universidad de Antioquia (Facultad de Ciencias Agrarias) para ser analizadas por expertos.

\section{Procesamiento histotécnico}

Los tejidos fueron fijados en formalina tamponada al $10 \%$ por 48 horas a $4^{\circ} \mathrm{C}$, incluidos en parafina, cortados a $4 \mu \mathrm{m}$ de espesor y coloreados con hematoxilinaeosina para ser lavados y almacenados en etanol:agua (75:25, v:v) de acuerdo con el método reportado por Vente-Spreeuwenberg et al. (2001). Estos cortes fueron 
microdiseccionados para determinar el promedio de la altura y ancho de las vellosidades intestinales, así como la profundidad y ancho de las criptas adyacentes. En cada lámina se montaron tres cortes transversales.

\section{EVALUACIÓN MICROSCÓPICA Y ANÁlISIS MORFOMÉTRICO DE IMÁGENES}

Los cortes histológicos fueron analizados cuantitativamente mediante un procesamiento de imágenes digitales computarizadas, así: para la identificación de las zonas tisulares se empleó un microscopio óptico Leica DLMB( Meyer, Houston, TX, USA), luego se capturaron las imágenes correspondientes con una cámara para microscopía digital instantánea Motican 2300 (Motic, Hong Kong, China) con una resolución de 3 megapixeles, en un aumento de $200 \times$ y se analizaron dichas imágenes con el software para tratamiento de imágenes Motic ${ }^{\circledR}$ Images plus 2.0 (Motic, Hong Kong, China).

Las variables morfométricas que se midieron en cada corte histológico fueron: vellosidades intestinales.

1. Altura: una vez se estableció la base de la vellosidad, desde su punto medio se trazó una línea hasta el ápice.

2. Ancho: con una línea se unieron los bordes apicales de las células epiteliales de lados opuestos, ubicadas aproximadamente en la mitad de la vellosidad.

También se determinó la profundidad y el ancho de las criptas intestinales, conforme a lo descrito previamente por (Marion et al., 2002).

\section{Diseño ESTADÍSTICO}

El experimento se realizó según un diseño bloques al azar ( 4 bloques: ubicación en el galpón: norte, sur, oriente y occidente; 4 bloques,) en un arreglo de parcelas dividas, donde los animales fueron aleatorizados a cada uno de los tratamientos (5 dietas por 7 períodos de evaluación), y cada tratamiento tuvo un total de 4 repeticiones. El análisis estadístico fue desarrollado usando el procedimiento GLM del SAS (2007).

\section{CONSIDERACIONES ÉTICAS}

Esta investigación fue avalada por el Comité de Ética en la Experimentación Animal de la Universidad Nacional de Colombia, Sede Medellín (CEMED 045 del 10 de junio de 2014).

\section{RESULTADOS}

En general las aves que consumieron las diferentes dietas exhibieron un buen estado de salud, y no presentaron enfermedades que causaran su retiro y/o sacrificio inmediato. Además al nivel en que se fijó el suministro diario de alimento no hubo sobrantes.

En este experimento no se encontró interacción estadística entre las diferentes dietas y los días de sacrificio para ninguna de las variables en estudio, por lo que no fue necesario analizar y desglosar dichos factores de manera independiente.
Tabla II. Peso de órganos (\% PV) y análisis alométrico (CA) de pollos que consumieron dietas con cepas probióticas (L. acidophilus, L. casei y E. faecium) durante 42 días (Organ weight (\%BW) and allometric analysis (CA) of broilers' fed on diets with probiotic strains (L. acidophilus, L. casei and $E$. faecium) during 42 days).

\begin{tabular}{|c|c|c|c|c|c|c|c|}
\hline \multirow{2}{*}{ Órgano } & \multicolumn{3}{|l|}{$(\% \mathrm{PV})$} & \multicolumn{3}{|c|}{ Dietas } & \multirow[b]{2}{*}{ EEM } \\
\hline & $\mathrm{CA}$ & D1 & D2 & D3 & D4 & D5 & \\
\hline \multirow{2}{*}{\multicolumn{2}{|c|}{ Proventrículo }} & $0,46^{A}$ & $0,51^{\mathrm{B}}$ & $0,51^{\mathrm{B}}$ & $0,52^{\mathrm{B}}$ & $0,56^{\mathrm{C}}$ & 0,003 \\
\hline & & $0,51^{\mathrm{A}}$ & $0,66^{\mathrm{B}}$ & $0,75^{\mathrm{C}}$ & $0,57^{\mathrm{D}}$ & $0,79^{\mathrm{E}}$ & 0,003 \\
\hline \multirow{2}{*}{\multicolumn{2}{|c|}{ Molleja }} & $1,98^{A}$ & $2,02^{\mathrm{A}}$ & $2,32^{\mathrm{B}}$ & $2,39^{c}$ & $2,47^{\mathrm{D}}$ & 0,015 \\
\hline & & $0,46^{A}$ & $0,56^{\mathrm{B}}$ & $0,46^{A}$ & $0,47^{A}$ & $0,57^{\mathrm{B}}$ & 0,005 \\
\hline \multirow{2}{*}{\multicolumn{2}{|c|}{ Páncreas }} & $0,23^{A}$ & $0,26^{\mathrm{B}}$ & $0,28^{\mathrm{B}}$ & $0,27^{\mathrm{B}}$ & $0,30^{\mathrm{C}}$ & 0,003 \\
\hline & & $0,36^{A}$ & $0,29^{\mathrm{B}}$ & $0,35^{\mathrm{A}}$ & $0,36^{A}$ & $0,42^{\mathrm{C}}$ & 0,008 \\
\hline \multirow{2}{*}{\multicolumn{2}{|c|}{ Hígado }} & $2,87^{A}$ & $2,97^{\mathrm{A}}$ & $3,24^{\mathrm{B}}$ & $3,19^{\mathrm{AB}}$ & $3,50^{c}$ & 0,040 \\
\hline & & $0,70^{A}$ & $0,82^{\mathrm{B}}$ & $0,69^{A}$ & $0,58^{\mathrm{C}}$ & $0,65^{\mathrm{D}}$ & 0,005 \\
\hline \multirow{2}{*}{\multicolumn{2}{|c|}{ Intestino }} & $5,92^{\mathrm{A}}$ & $6,30^{\mathrm{B}}$ & $6,77^{\mathrm{C}}$ & $6,60^{\mathrm{C}}$ & $6,91^{\mathrm{D}}$ & 0,027 \\
\hline & & $0,45^{A}$ & $0,46^{\mathrm{A}}$ & $0,48^{\mathrm{A}}$ & $0,53^{\mathrm{C}}$ & $0,56^{\mathrm{D}}$ & 0,004 \\
\hline
\end{tabular}

D1= Alimento comercial sin probiótico y sin antibiótico; D2=Alimento comercial + antibiótico; D3= Alimento comercial sin antibiótico $+L$. acidophilus; D4=Alimento comercial sin antibiótico + L. casei; D5=Alimento comercial sin antibiótico + E. faecium; \% PV=Peso órgano $\times 100 /$ (Peso promedio/ave); $\mathrm{CA}=\left(\mathrm{O}_{\mathrm{n}} / \mathrm{O}_{h}\right) /\left(\mathrm{PC}_{\mathrm{n}} / \mathrm{PC}_{h}\right)$, donde: $\mathrm{O}=$ peso del órgano; $\mathrm{n}=$ días después del nacimiento; $\mathrm{h}=$ peso al nacimiento $\mathrm{y}$ $\mathrm{PC}=$ peso corporal. $(\mathrm{CA}<1)=$ Crecimiento lento con relación al peso corporal; $(C A=1)=$ Crecimiento proporcional con relación al peso corporal. $(C A>1)=$ Crecimiento rápido con relación al peso corporal. $A, B, C, D$ Dentro de una misma fila medias con un superíndice común no difieren estadísticamente $(p<0,05)$; EEM= Error estándar de la media.

Para la variable peso de órganos (\% PV) de proventrículo, páncreas e intestino se presentaron diferencias significativas entre D1 y D2, y entre D2 y D5 ( $<<0,05$; tabla II); entre D2, D3 y D4 no se presentaron diferencias significativas. Para molleja e hígado no se presentaron diferencias entre D1 y D2, pero entre D2 y D3, D4 y D5 hubo diferencias significativas $(p<0,05)$.

Para la variable crecimiento alométrico (CA) se presentaron diferencias significativas ( $p<0,05$; tabla II) entre todas las dietas para proventrículo. Para molleja y páncreas se presentaron diferencias entre D1 y D2, y entre D2, D3 y D4 $(\mathrm{p}<0,05)$, y entre D2 y D5 no se presentaron diferencias. Para hígado se presentaron diferencias entre D1 y D2, y entre D2, D3, D4 y D5 $(p<0,05)$. Para intestino entre D1, D2 y D3, no se presentaron diferencias significativas, mientras que entre $\mathrm{D} 2$, D4 y D5 se presentaron diferencias $(\mathrm{p}<0,05)$. Todos los órganos presentaron un crecimiento lento $(\mathrm{CA}<1)$ con relación al peso corporal (tabla II).

En el análisis alométrico de órganos en los diferentes días de sacrificio se presentaron diferencias estadísticamente significativas ( $p<0,05)$, obteniéndose que en la evaluación realizada el día 42 las aves presentaron los mayores valores para cada uno de los órganos, mientras que los menores valores se obtuvieron en la primera evaluación (día 14) (tabla III). Todos los órganos presentan un crecimiento lento $(\mathrm{CA}<1)$ en relación con el peso corporal en los diferentes períodos de tiempo (tabla III). 
Para la variable altura de vellosidades, se presentó diferencia significativa $(\mathrm{p}<0,05)$ entre los animales que consumieron D1 y D2, donde la sección intestinal duodeno presentó los mayores valores para D2. No obstante al comparar D2 con las demás dietas, D5 presentó los mayores valores $(\mathrm{p}<0,05)$ para duodeno, yeyuno e íleon. Para la variable ancho de vellosidades,

Tabla III. Peso de órganos (\% PV) y análisis alométrico (CA) de pollos que consumieron cepas probióticas (L. acidophilus, L. casei y E. faecium) durante diferentes períodos de tiempo (Organ weight (\% PV) and allometric analysis (CA) of broilers' fed on diets with probiotic strains (L. acidophilus, L. casei and E. faecium) during several time periods).

\begin{tabular}{|c|c|c|c|c|c|c|c|c|}
\hline \multirow{2}{*}{ Órgano } & \multicolumn{3}{|c|}{$(\% \mathrm{PV})$} & \multicolumn{4}{|c|}{ Días } & \multirow[b]{2}{*}{ EEM } \\
\hline & $\mathrm{CA}$ & 7 & 14 & 21 & 28 & 35 & 42 & \\
\hline \multirow{2}{*}{\multicolumn{2}{|c|}{ Proventrículo }} & $0,56^{\mathrm{A}}$ & $0,64^{\mathrm{C}}$ & $0,89^{c}$ & $0,91^{\mathrm{D}}$ & $0,71^{\mathrm{E}}$ & $0,83^{F}$ & 0,003 \\
\hline & & - & $0,50^{A}$ & $056^{c}$ & $0,66^{c}$ & $0,78^{\mathrm{D}}$ & $0,82^{\mathrm{E}}$ & 0,00 \\
\hline \multirow{2}{*}{\multicolumn{2}{|c|}{ Molleja }} & $3,02^{\mathrm{A}}$ & $3,54^{\mathrm{C}}$ & $3,67^{\mathrm{c}}$ & $3,64^{\mathrm{D}}$ & $2,78^{\mathrm{E}}$ & $2,96^{\mathrm{F}}$ & 0,015 \\
\hline & & - & $0,36^{A}$ & $0,37^{\mathrm{A}}$ & $0,49^{c}$ & $0,63^{\mathrm{D}}$ & $0,80^{\mathrm{E}}$ & 0,005 \\
\hline \multirow{2}{*}{\multicolumn{2}{|c|}{ Páncreas }} & $0,49^{A}$ & $0,29^{c}$ & $0,51^{\mathrm{C}}$ & $0,42^{\mathrm{D}}$ & $0,30^{\mathrm{E}}$ & $0,40^{F}$ & 0,003 \\
\hline & & - & $0,24^{\mathrm{A}}$ & $0,31^{\mathrm{c}}$ & $0,34^{\mathrm{C}}$ & $0,40^{\mathrm{C}}$ & $0,54^{\mathrm{D}}$ & 0,008 \\
\hline \multirow{2}{*}{\multicolumn{2}{|c|}{ Hígado }} & $3,33^{A}$ & $4,21^{\mathrm{C}}$ & $4,95^{\mathrm{C}}$ & $5,45^{\mathrm{D}}$ & $5,33^{\mathrm{E}}$ & $4,77^{F}$ & 0,040 \\
\hline & & - & $0,54^{\mathrm{A}}$ & $0,62^{\mathrm{C}}$ & $0,66^{\mathrm{c}}$ & $0,77^{\mathrm{D}}$ & $0,86^{\mathrm{E}}$ & 0,005 \\
\hline \multirow{2}{*}{\multicolumn{2}{|c|}{ Intestino }} & $8,95^{\mathrm{A}}$ & $6,75^{\mathrm{C}}$ & $10,91^{\mathrm{C}}$ & $11,30^{\mathrm{D}}$ & $9,62^{\mathrm{E}}$ & $10,33^{F}$ & 0,027 \\
\hline & & - & $0,51^{\mathrm{A}}$ & $0,43^{c}$ & $0,51^{\mathrm{A}}$ & $0,42^{\mathrm{c}}$ & $0.63^{c}$ & 0,004 \\
\hline
\end{tabular}

$\% \mathrm{PV}=$ Peso órgano $\times 100 /($ Peso promedio/ave $) ; \mathrm{CA}=\left(\mathrm{O}_{\mathrm{n}} / \mathrm{O}_{\mathrm{h}}\right) /\left(\mathrm{PC}_{\mathrm{n}} /\right.$ $\mathrm{PC}_{\mathrm{h}}$ ), donde: $\mathrm{O}=$ peso del órgano; $\mathrm{n}=$ días después del nacimiento; $\mathrm{h}=$ peso al nacimiento y $\mathrm{PC}=$ peso corporal. $(\mathrm{CA}<1)=$ Crecimiento lento con relación al peso corporal; $(C A=1)=$ Crecimiento proporcional con relación al peso corporal. $(C A>1)=$ Crecimiento rápido con relación al peso corporal. ${ }^{A, B, C, D}$ Dentro de una misma fila medias con un superíndice común no difieren estadísticamente $(p<0,05)$; $E E M=$ Error estándar de la media. se presentó diferencia $(\mathrm{p}<0,05)$ entre D1 y D2, obteniéndose los mayores valores para D2 en las tres porciones del intestino; sin embargo, al comparar D2 con las demás dietas, se encontraron diferencias significativas $(\mathrm{p}<0,05)$, donde D5 reporta los mayores valores para duodeno, yeyuno e íleon (tabla IV).

Para la variable profundidad de criptas se presentó diferencia significativa $(\mathrm{p}<0,05)$ entre D1 y D2, donde D2 presenta valores menores (tabla IV). D5 presentó los valores más bajos $(\mathrm{p}<0,05)$ en comparación con las demás dietas en todas las porciones del intestino. Para la variable ancho de cripta (tabla IV) D1 presentó los mayores valores $(p<0,05)$ en comparación con D2. En las demás porciones del intestino D5 presentó los valores más bajos $(\mathrm{p}<0,05)$.

Al comparar las porciones del intestino, también se presentaron diferencias significativas $(p<0,05)$ en la altura y el ancho de las vellosidades. En cada una de las dietas duodeno presentó una mayor altura de vellosidades en comparación con yeyuno e íleon. Además, para las variables profundidad y ancho de criptas se encontró que el duodeno presentó menor profundidad y ancho de criptas en comparación con yeyuno e íleon, lo cual se observó en todas las dietas (tabla IV).

Con la inclusión de bacterias acido lácticas en dietas de pollos de engorde durante 42 días, se presentaron diferencias significativas $(\mathrm{p}<0,05)$ en los tres períodos evaluados. En el día 1 las aves presentaron menor altura y ancho de vellosidades en cada una de las porciones del intestino y las criptas presentaron mayor profundidad y mayor ancho; al día 21 y 42 se observa como la altura y el ancho de las vellosidades aumentan, y la profundidad y ancho de las criptas disminuye (tabla V). También se observa que en cada periodo

Tabla IV. Comparación entre vellosidades y criptas $(\mu \mathrm{m})$ en diferentes secciones del intestino de pollos que consumieron dietas con cepas probióticas (L. acidophilus, L. casei y E. faecium) durante 42 días (Comparison between villi and crypts $(\mu \mathrm{m})$ in different sections of the intestine of broilers' fed on diets with different probiotic strains (L. acidophilus, $L$. casei and $E$. faecium) during 42 days).

\begin{tabular}{|c|c|c|c|c|c|c|c|}
\hline \multirow{2}{*}{ Variable $(\mu \mathrm{m})$} & & \multicolumn{5}{|c|}{ Dietas } & \multirow[b]{2}{*}{ EEM } \\
\hline & & D1 & D2 & D3 & D4 & D5 & \\
\hline \multicolumn{8}{|c|}{ Vellosidades $(\mu \mathrm{m})$} \\
\hline & Duodeno & $742,4^{\mathrm{AX}}$ & $805,2^{\mathrm{BX}}$ & $884,3^{c x}$ & $921,6^{\mathrm{Dx}}$ & $996,1^{\mathrm{EX}}$ & \\
\hline \multirow[t]{3}{*}{ Altura } & Yeyuno & $708,1^{\mathrm{AX}}$ & $779,3^{\mathrm{BY}}$ & $857,5^{\mathrm{CY}}$ & $904,6^{\mathrm{DXY}}$ & $965,4^{\mathrm{EY}}$ & 3,14 \\
\hline & Íleon & $691,9^{A Y}$ & $764,4^{\mathrm{BY}}$ & $839,6^{\mathrm{CY}}$ & $884,2^{\mathrm{DY}}$ & $935,3^{\mathrm{EZ}}$ & \\
\hline & Duodeno & $121,3^{\mathrm{A}}$ & $132,4^{\mathrm{BX}}$ & $145,1^{\mathrm{cx}}$ & $156,6^{\mathrm{DX}}$ & $164,0^{\mathrm{EX}}$ & \\
\hline \multirow[t]{2}{*}{ Anchura } & Yeyuno & $119,4^{\mathrm{A}}$ & $126,7^{\mathrm{BXY}}$ & $137,2^{\mathrm{CY}}$ & $148,3^{D Y}$ & $157,3^{\mathrm{EY}}$ & 1,09 \\
\hline & Íleon & $117,2^{\mathrm{A}}$ & $122,2^{\mathrm{BY}}$ & $132,7^{\mathrm{CY}}$ & $145,7^{\mathrm{DY}}$ & $155,1^{\mathrm{EZ}}$ & \\
\hline \multicolumn{8}{|c|}{ Criptas $(\mu \mathrm{m})$} \\
\hline \multirow{3}{*}{ Profundidad } & Duodeno & $119,4^{\mathrm{AX}}$ & $114,2^{\mathrm{BX}}$ & $109,4^{c x}$ & $107,8^{c x}$ & $103,5^{\mathrm{Dx}}$ & \\
\hline & Yeyuno & $125,2^{\mathrm{AY}}$ & $121,5^{\mathrm{BY}}$ & $117,4^{\mathrm{CY}}$ & $112,6^{\mathrm{DY}}$ & $107,4^{\mathrm{EY}}$ & 0,61 \\
\hline & Íleon & $128,3^{A Y}$ & $124,6^{\mathrm{BY}}$ & $119,1^{\mathrm{Cr}}$ & $114,7^{\mathrm{DY}}$ & $109,6^{\mathrm{EY}}$ & \\
\hline \multirow{3}{*}{ Anchura } & Duodeno & $111,9^{A X}$ & $107,5^{\mathrm{BX}}$ & $104,5^{c x}$ & $102,8^{\mathrm{CX}}$ & $99,4^{\mathrm{Dx}}$ & \\
\hline & Yeyuno & $112,1^{\mathrm{AY}}$ & $109,2^{\mathrm{BY}}$ & $106,2^{\mathrm{CY}}$ & $106,1^{\mathrm{CY}}$ & $103,2^{D Y}$ & 0,38 \\
\hline & Íleon & $113,2^{\mathrm{AZ}}$ & $110,3^{B Z}$ & $107,5^{\mathrm{cz}}$ & $107,1^{\mathrm{CDY}}$ & $106,7^{c z}$ & \\
\hline
\end{tabular}

D1= Alimento comercial sin probiótico y Sin antibiótico; D2= Alimento comercial + antibiótico; D3= Alimento comercial sin antibiótico + L. acidophilus; D4= Alimento comercial sin antibiótico + L. casei; D5= Alimento comercial sin antibiótico + E. faecium. A,B,C,D,EDentro de una misma fila (horizontal) medias con un superíndice común no difieren estadísticamente $(p<0,05)$; $x, Y, Z$ Dentro de una misma columna (vertical) medias con un superíndice común no difieren estadísticamente $(p<0,05)$; EEM= Error estándar de la media. 
Tabla V. Comparación entre vellosidades y criptas $(\mu \mathrm{m})$ en diferentes secciones del intestino de pollos que consumieron cepas probióticas (L. acidophilus, L. casei y E. faecium) durante diferentes períodos de tiempo (Comparison between villi and crypts $(\mu \mathrm{m})$ in different sections of the intestine of broilers' fed on diets with different probiotic strains (L. acidophilus, $L$. casei and E. faecium) during several time periods).

\begin{tabular}{|c|c|c|c|c|c|}
\hline \multirow[t]{2}{*}{ Variable } & & \multicolumn{3}{|c|}{ Días } & \multirow{2}{*}{ EEM } \\
\hline & & 1 & 21 & 42 & \\
\hline \multicolumn{6}{|c|}{ Vellosidades $(\mu \mathrm{m})$} \\
\hline & Duodeno & $779,5^{\mathrm{AX}}$ & $869,3^{\mathrm{BX}}$ & $961,1^{\mathrm{cx}}$ & \\
\hline \multirow[t]{3}{*}{ Altura } & Yeyuno & $753,7^{\mathrm{AY}}$ & $834,9^{\mathrm{BY}}$ & $939,3^{\mathrm{Cr}}$ & 2,84 \\
\hline & Íleon & $735,6^{\mathrm{AY}}$ & $820,4^{B Z}$ & $912,3^{\mathrm{cz}}$ & \\
\hline & Duodeno & $126,7^{\mathrm{AX}}$ & $144,7^{\mathrm{BX}}$ & $159,1^{\mathrm{cx}}$ & \\
\hline \multirow[t]{2}{*}{ Anchura } & Yeyuno & $120,9^{A Y}$ & $143,2^{\mathrm{BXY}}$ & $149,3^{\mathrm{BY}}$ & 1,54 \\
\hline & Íleon & $119,1^{\mathrm{AY}}$ & $136,7^{\mathrm{BY}}$ & $147,8^{\mathrm{Cr}}$ & \\
\hline \multicolumn{6}{|c|}{ Criptas $(\mu \mathrm{m})$} \\
\hline \multirow{3}{*}{ Profundidad } & Duodeno & $116,5^{\mathrm{AX}}$ & $110,5^{\mathrm{BX}}$ & $105,3^{c x}$ & \multirow{3}{*}{0,81} \\
\hline & Yeyuno & $123,5^{\mathrm{AY}}$ & $116,1^{\mathrm{BY}}$ & $111,2^{\mathrm{CY}}$ & \\
\hline & Íleon & $125,4^{\mathrm{AY}}$ & $120,7^{\mathrm{BY}}$ & $112,1^{\mathrm{CY}}$ & \\
\hline \multirow{3}{*}{ Anchura } & Duodeno & $109,5^{\mathrm{A}}$ & $106,5^{\mathrm{B}}$ & $101,2^{\mathrm{cx}}$ & \multirow{3}{*}{0,45} \\
\hline & Yeyuno & $110,5^{A}$ & $107,2^{\mathrm{B}}$ & $104,5^{\mathrm{CY}}$ & \\
\hline & Íleon & $111,6^{A}$ & $108,7^{\mathrm{B}}$ & $106,1^{\mathrm{BY}}$ & \\
\hline
\end{tabular}

$\mathrm{A}, \mathrm{B}, \mathrm{C}, \mathrm{D}, \mathrm{E}$ Dentro de una misma fila (horizontal) medias con un superíndice común no difieren estadísticamente $(p<0,05)$; ${ }^{x, Y, Z}$ Dentro de una misma columna (vertical) medias con un superíndice común no difieren estadísticamente $(p<0,05)$; EEM= Error estándar de la media.

de tiempo evaluado si se comparan las porciones del intestino entre ellas, el duodeno presenta mayor altura $\mathrm{y}$ ancho de vellosidades $\mathrm{y}$ menor profundidad $\mathrm{y}$ ancho de criptas, en comparación con yeyuno e íleon.

\section{DISCUSIÓN}

En este trabajo se encontraron mayores pesos de órganos en las aves alimentadas con cepas probióticas, específicamente con E. faecium, en comparación con la dieta que contenía antibiótico (D2), constatando que con la utilización de probióticos se incrementa la retención de nutrientes incluidos en la dieta, la ganancia de peso y el desarrollo de órganos del animal (Franz et al., 2011). Las líneas genéticas de pollos utilizadas actualmente tienen un crecimiento más rápido, por lo que necesitan que sus órganos tengan un desarrollo acelerado, lo que se obtuvo en los animales que consumieron probióticos, específicamente E. faecium.

Durante la primera semana de vida del ave se da un crecimiento rápido de los órganos, coincidiendo con lo reportado por Cuervo et al. (2002), donde el peso de órganos digestivos como intestino, hígado, páncreas, molleja y proventrículo aumentan significativamente durante la primera semana de vida, lo que conlleva al aumento de los procesos digestivos, y por ende, en el comportamiento productivo del ave. La inclusión de microorganismos probióticos en la dieta de pollos de engorde permite el rápido desarrollo de las bacterias benéficas en el tracto digestivo, mejorando su rendimiento. Como consecuencia, hay una mejora en el ambiente intestinal, aumentando la eficiencia de los procesos de digestión y absorción de nutrientes, los cuales se ven reflejados en las ganancias de peso y por consiguiente en el desarrollo de los órganos (Alkhalf et al., 2010).

Según Svihus (2011), cuando se añaden a la dieta componentes que mejoran la disponibilidad de nutrientes, como en este caso los probióticos, se estimula el crecimiento y desarrollo de molleja y proventrículo. Lo anterior concuerda con los encontrados en este trabajo, donde proventrículo y molleja, presentaron el mayor crecimiento durante los días 28 y 42 en comparación con el resto de días. El páncreas mostró un crecimiento alométrico lento en relación con el peso corporal en cada uno de los tratamientos; sin embargo, el páncreas es un órgano pequeño e inmaduro funcionalmente durante los primeros días de vida del ave, pero luego sufre una rápida maduración debido a la actividad de las enzimas pancreáticas que ayudan a la digestión de carbohidratos, lípidos y proteínas (Cuervo et al., 2002). No obstante, el intestino mostró un crecimiento lento en relación al peso corporal en esta investigación, lo que concuerda con Jaramillo (2012) quien encontró que durante la primera y segunda semana después de la eclosión, el intestino muestra una disminución en el tamaño relativo al peso corporal, lo que podría comprometer directamente la salud intestinal, la absorción de nutrientes, y por ende el crecimiento y desarrollo del ave. La diferencia de CA del hígado entre las diferentes dietas coincide con lo reportado por Anwar et al. (2012), donde encontraron que aves suplementadas con probióticos tenían menor CA comparado con aves alimentadas sin aditivos probióticos, lo que puede estar relacionado con una mayor movilización de grasas y glucosa a partir del hígado.

El consumo de probióticos ha sido asociada a mejoras en parámetros productivos, los cuales influyen sobre el peso y desarrollo de órganos, específicamente del intestino (Franz et al., 2011). En este trabajo se encontró que las aves alimentadas con E. faecium presentaron mayor desarrollo intestinal reflejado en vellosidades con mayor altura y ancho, y criptas menos profundas (Franz et al., 2011). En pollos, la altura de las vellosidades intestinales aumenta y la profundidad de las criptas disminuye rápidamente tras la eclosión, lo que incrementa la superficie de absorción de nutrientes. Las aves alimentadas con la dieta basal presentaron criptas más profundas y más anchas, lo que implica un mayor requerimiento por el alto recambio celular para el mantenimiento de este tejido (Giannenas et al., 2012; Salim et al., 2013; Yu et al., 2011). Le Bon et al. (2010), reportaron que la adicion de probioticos en el alimento de lechones destetos durante 9 semanas tuvo una influencia positiva sobre la salud e integridad intestinal, lo cual se vió reflejado en la disminución de los niveles de coliformes. Cao et al. (2013), evaluaron la inclusion de E. faecium en pollos de engorde y encontraron que a nivel intestinal las vellosidades presentaron mayor altura y la profundidad de criptas fue menor, en comparación con el control, lo que esta asociado al aumento en la eficiencia de crecimiento y en la superficie de absorción de nutrientes; coincidiendo con lo reportado en este estudio. 
Deng et al. (2012) reportaron que la inclusión de probioticos en el alimento de gallinas de postura sometidas a estrés calorico presentaban una mejor estructura intestinal (vellosidades mas altas y criptas menos profundas). Fallah et al. (2013) y Al-Fataftah and Abdelqader (2014) incluyeron en la alimentación de pollos de engorde un probiotico comercial, obteniendo a nivel de duodeno y yeyuno vellosidades mas altas, favoreciendo la absorción de nutrientes y por ende, el crecimiento y desarrollo del animal. Todo lo anterior concuerda con los datos obtenidos en esta investigación. Markovic et al. (2009) reportaron que la adición de probioticos en la alimentación de pollos de engorde proporciona una microflora intestinal mas estable, la cual mejora las condiciones para la vida util de los enterocitos, disminuye la renovacion celular intestinal y demanda de nutrientes (Awad et al., 2009), e influencia el estado inmune y salud general del animal (Rajput $e t$ al., 2013; Lee et al., 2014).

El consumo de probióticos ha sido asociado a mejoras en el peso y desarrollo del intestino (Franz et al., 2011), debido a que favorecen el crecimiento de las vellosidades intestinales, y a su vez, aumentan la actividad enzimática, el transporte y la absorción de nutrientes en el epitelio intestinal (Giang et al., 2010). En la literatura se ha reportado que los probióticos pueden minimizar la proliferación de agentes patógenos al competir por nutrientes y sitios de adhesión en las paredes intestinales, disminuir la actividad de las enzimas bacterianas y la producción de amoniaco, neutralizar enterotoxinas y estimular el sistema inmune (Musa et al., 2009). Asímismo, poseen mecanismos no inmunológicos que mejoran la estabilización de la barrera de intestinal, la secreción de moco, y la motilidad intestinal (Giang et al., 2010; Tsirtsikos et al., 2012).

\section{CONCLUSIONES}

En este estudio, la inclusión de probióticos en la alimentación de pollos de engorde, aumentaron el peso, desarrollo y crecimiento de órganos de importancia digestiva, específicamente intestino, lo cual se vió reflejado en vellosidades más altas y criptas menos profundas, lo que podría mejorar la absorción de nutrientes y por consiguiente la salud de los animales. Por lo anterior, E. faecium, puede ser utilizado en la alimentación de aves durante todo el ciclo productivo como promotor de crecimiento.

\section{BIBLIOGRAFÍA}

Al-Fataftah, A.R. and Abdelqader, A. 2014. Effects of dietary Bacillus subtilis on heat-stressed broilers performance, intestinal morphology and microflora composition. Anim Feed Sci Technol, 198: 279-285.

Alkhalf, A.; Alhaj, M. and Al-Homidan, I. 2010. Influence of probiotic supplementation on blood parameters and growth performance in broiler chickens. Saudi J Biol Sci, 17: 219-225.

Anwar, H.; Rahman, Z.; Javed, I. and Muhammad, F. 2012. Efficacy of protein, symbiotic and probiotic supplementation on body performance and organs weight in molted layers. Pak Vet J, 33: 117-119.
Awad, W.A.; Ghareeb, K.; Abdel-Raheem, S. and Böhm, J. 2009. Effects of dietary inclusion of probiotic and synbiotic on growth performance, organ weights, and intestinal histomorphology of broiler chickens. Poult Sci, 88: 49-56.

Cao, G.T.; Zeng, X.F.; Chen, A.G.; Zhou, L.; Zhang, L.; Xiao, Y.P. and Yang, C.M. 2013. Effects of a probiotic, Enterococcus faecium, on growth performance, intestinal morphology, immune response, and cecal microflora in broiler chickens challenged with Escherichia coli K88. Poult Sci, 92: 2949-2955.

Chambers, J.R. and Gong, J. 2011 . The intestinal microbiota and its modulation for salmonella control in chickens. Food Res Int, 44: 31 49-3159.

Cortés, L.y Villamarín, S. 2013. Características morfométricas de órganos linfoides y estudios serológicos en levante de ponedoras utilizando un inmunomodulador, vitaminas y aminoácidos. Spei Domus, 9: 29-36.

Cuervo, M.; Gómez, C. y Romero, H. 2002. Efecto de la utilización de un suplemento nutricional hidratado en pollos de engorde recién nacidos. Rev Colomb Cienc Pec, 15: 319-329.

Deng, W.; Dong, X.F.; Tong, J.M. and Zhang, Q. 2012. The probiotic Bacillus licheniformis ameliorates heat stress-induced impairment of egg production, gut morphology, and intestinal mucosal immunity in laying hens. Poult Sci, 91: 575-582.

Dolz, R. and Majó, N. 2011. Atlas de necropsia aviar. Servet. Zaragoza. España.

Fallah, R.; Saghafi, M.; Rezaei, H. and Parvar, R. 2013. Effect of Bioplus $2 B \otimes$ and protoxin probiotics supplementation on growth performance, small intestinal morphology and carcass characteristics of broiler chickens. Brit J Poult Sci, 2: 11-15.

Franz, C.M.; Huch, M.; Abriouel, H.; Holzapfel, W. and Gálvez, A. 2011 . Enterococci as probiotics and their implications in food safety. Int J Food Microbiol, 151: 125-140.

Gaggia, F.; Mattarelli, P. and Biavati, B. 2010. Probiotics and prebiotics in animal feeding for safe food production. Int J Food Microbiol, 141 Suppl: S15-28.

Giang, H.H.; Viet, T.Q.; Ogle, B. and Lindberg, J.E. 2010. Growth performance, digestibility, gut environment and health status in weaned piglets fed a diet supplemented with potentially probiotic complexes of lactic acid bacteria. Livest Sci, 129: 95-103.

Giannenas, I.; Papadopoulos, E.; Tsalie, E.; Triantafillou, E.; Henikl, S.; Teichmann, K. and Tontis, D. 2012. Assessment of dietary supplementation with probiotics on performance, intestinal morphology and microflora of chickens infected with Eimeria tenella. Vet Parasitol, 188: 31-40.

Huyghebaert, G.; Ducatelle, R. and Van Immerseel, F. 2011 . An update on alternatives to antimicrobial growth promoters for broilers. Vet J, 187: 182-188.

Jaramillo, A. 2012. Evaluación de la mezcla de un ácido orgánico y un prebiótico en los parámetros productivos y alométricos de pollos de engorde con alimentación controlada. Rev Colombiana Cienc Anim, 5: 52-66.

Kalmar, I.D.; Vanrompay, D. and Janssens, G.P.J. 2013. Broiler ascites syndrome: collateral damage from efficient feed to meat conversion. Vet J, 197: 169-174.

Korver, D.R. 2012. Implications of changing immune function through nutrition in poultry. Anim Feed Sci Technol, 173: 54-64.

Král, M.; Angelovičová, M. and Mrázová, L'. 2012. Application of probiotics in poultry production. Anim Sci Biotechnol, 45: 55-58.

Le Bon, M.; Davies, H.E.; Glynn, C.; Thompson, C.; Madden, M.; Wiseman, J.; Dodd, C., Hurdidge, L.; Payne, G.; Le Treut, Y.; Craigon, J.; Tötemeyer, S. and Mellits, K.H. 2010. Influence of probiotics on gut health in the weaned pig. Livest Sci, 133: 179-181.

Lee, S.H.; Ingale, S.L.; Kim, J.S.; Kim, K.H.; Lokhande, A.; Kim, E.K.; Kwon, E.K.; Kim, Y.H. and Chae, B.J. 2014. Effects of dietary supplementation with Bacillus subtilis LS 1-2 fermentation biomass on growth performance, nutrient digestibility, cecal microbiota and intestinal morphology of weanling pig. Anim Feed Sci Technol, 188: 102-110. Lodemann, U. 2010. Effects of probiotics on intestinal transport and epithelial barrier function. In: R.R. Watson and V. Preedy (Eds.). 
Bioactive foods in promoting health: probiotics and prebiotics. $1^{\text {st }} \mathrm{ed}$. pp. 303-333.

Marion, J.; Biernat, M.; Thomas, F.; Savary, G.; Le Breton, Y.; Zabielski, R.; Le Huërou-Luron, I. and Le Dividich, J. 2002. Small intestine growth and morphometry in piglets weaned at 7 days of age. effects of level of energy intake. Reprod Nutr Dev, 42: 339-354.

Markovic, R.; Šefer, D.; Krstic, M. and Petrujkic, B. 2009. Effect of different growth promoters on broiler performance and gut morphology. Arch Med Vet, 41: 163-169.

Musa, H.H.; Wu, S.L.; Zhu, C.H.; Seri, H.I. and Zhu, G.Q. 2009. The potential benefits of probiotics in animal production and health. $J$ Anim Vet Adv, 8: 313-321.

Plaza, J.; Gomez, C.; Fontana, L. and Gil, A. 2014. Modulation of immunity and inflammatory gene expression in the gut, in inflammatory diseases of the gut and in the liver by probiotics. World J Gastroenterol, 20: 15632-15649.

Quigley, E.M.M. 2010. Prebiotics and probiotics; modifying and mining the microbiota. Pharmacol Res, 61: 213-218.

Rajput, I.R.; Li, L.Y.; Xin, X.; Wu, B.B.; Juan, Z.L.; Cui, Z.W.; Yu, D.Y. and Li, W.F. 2013. Effect of Saccharomyces boulardii and Bacillus subtilis B10 on intestinal ultrastructure modulation and mucosal immunity development mechanism in broiler chickens. Poult Sci, 92: 956-965.
Reis, S.T.C.; Guerrero, C.M.J.; Aguilera, B.A. y Mariscal, L.G. 2005. Efecto de diferentes cereales sobre la morfología intestinal de lechones recién destetados. Téc Pecu Mex, 43: 309-321.

Salim, H.M.; Kang, H.K.; Akter, N.; Kim, D.W.; Kim, J.H.; Kim, M.J.; Na, J.C.; Jong, H.B.; Choi, H.C.; Suh, O.S. and Kim, W.K. 2013. Supplementation of direct-fed microbials as an alternative to antibiotic on growth performance, immune response, cecal microbial population, and ileal morphology of broiler chickens. Poult Sci, 92: 2084-2090.

Svihus, B. 2011 . La molleja: influencia de la estructura de la dieta y efectos sobre la disponibilidad de nutrientes. WPS J, 67: 1-11.

Tsirtsikos, P.; Fegeros, K.; Balaskas, C.; Kominakis, A. and Mountzouris, K.C. 2012. Dietary probiotic inclusion level modulates intestinal mucin composition and mucosal morphology in broilers. Poult Sci, 91: 1860-1868.

Vente-Spreeuwenberg, M.A.M.; Verdonk, A.C.; Gaskins, H.R. and Verstegen, M.W.A. 2001. Small intestine epithelial barrier function is compromised in pigs with low feed intake at weaning. J Nutr, 131: 1520-1527.

Yu, X.Y.; Yin, H.H. and Zhu, J.C. 2011 . Increased gut absorptive capacity in rats with severe head injury after feeding with probiotics. Nutrition, 27: 100-107. 